\title{
El empleo de videotutoriales en la era post COVID19: valoración e influencia en la identidad docente del futuro profesional.
}

\section{The use of video tutorials in the post-COVID19 era: assessment and influence on the development of the teaching identity of the future education professional.}

\author{
Juan Pablo Hernández-Ramos \\ Universidad de Salamanca. Salamanca, España \\ juanpablo@usal.es \\ Fernando Martínez-Abad \\ Universidad de Salamanca. Salamanca, España \\ fmausal.es \\ José Carlos Sánchez-Prieto \\ Universidad de Salamanca. Salamanca, España \\ josecarlos.sp@usal.es
}

\begin{abstract}
RESUMEN
Debido a la pandemia del COVID19, en el curso 2019-2020 en infinidad de países, se tuvo que suspender la actividad educativa presencial en cualquiera de sus manifestaciones, teniendo los docentes que planificar y desarrollar un nuevo proceso de enseñanza-aprendizaje virtual. Ante esta circunstancia y a tenor de la incertidumbre actual, los recursos tecnológicos se convierten en un aliado para el profesorado, siendo necesaria la difusión de experiencias como la recogida en este estudio: el empleo de videotutoriales en la enseñanza superior. A partir de un diseño de investigación cuasi-experimental solo postest de un grupo, tras aplicar un cuestionario electrónico a 240 estudiantes, se analiza la planificación e implementación de videotutoriales para la transmisión de los contenidos teóricos y prácticos en asignaturas de Metodología de Investigación. Las valoraciones realizadas muestran un nivel alto de aceptación del recurso, especialmente para: analizar y reflexionar sobre los contenidos; resolver problemas prácticos y organizar el estudio. Así mismo, queda reflejada la relación entre la percepción de utilidad del videotutorial y la intención de uso futuro. Se concluye el estudio destacando las potencialidades educativas del empleo de videotutoriales en la enseñanza superior a distancia.
\end{abstract}

Palabras clave: Video educativo, tecnología educativa, Educación superior, Transformación digital, COVID-19

\begin{abstract}
Due to the COVID19 pandemic, in the 2019-2020 school year in many countries, the classroom activity had to be suspended in any of its manifestations, having the teachers to plan and develop a new process of virtual teaching-learning. In view of this circumstance and in light of the current uncertainty, technological resources become an ally for teachers, making it necessary to disseminate experiences such as the one collected in this study: the use of video tutorials in higher education. From a quasi-
\end{abstract}


experimental only posttest single group research design, after applying an electronic questionnaire to 240 students, the planning and implementation of video tutorials for the transmission of theoretical and practical contents in Research Methodology subjects is analyzed. The assessments made show a high level of acceptance of the resource, especially for: analyzing and reflecting on the contents; solving practical problems and organizing the study. Likewise, the relationship between the perception of the usefulness of the video tutorial and the intention of future use is reflected. The study is concluded by highlighting the educational potential of the use of video tutorials in distance higher education

Keywords: Educational video, educational technology, Higher education, Digital transformation, COVID-19

\section{INTRODUCCIÓN}

La crisis sanitaria provocada por la irrupción del coronavirus (SARS-CoV-2) ha alterado la forma de vida de todo el mundo, generando retos en todos los ámbitos, sin ser el educativo una excepción. En el informe elaborado por Reimers y Schleider (2020) para la Organización para la Cooperación y el Desarrollo Económico (OCDE), recogen como casi la totalidad de las actuaciones de los distintos países se han centrado en desarrollar planes para la continuación de los procesos de enseñanza mediante modalidades alternativas. En la enseñanza universitaria, la necesidad de suprimir todas las actividades presenciales ha generado una transición forzosa de una enseñanza presencial a una digital para concluir el curso 2019-2020; convirtiéndose en el momento de la historia reciente en que la tecnología ha tenido mayor demanda por parte de la educación (Mateo-Berganza \& Lee, 2020).

En la actualidad, en base a la incertidumbre por la evolución de la pandemia, en todos los contextos educativos los profesionales se preguntan cómo será la enseñanza en los próximos cursos. Además, estudios como el realizado por Trujillo-Sáez y colaboradores (2020), apoyan la idea de que es el momento adecuado para repensar nuestro modelo educativo por completo. Por tanto, el desafío actual, de cara a una educación superior de calidad, no solo reside en superar las adversidades originadas por la pandemia del COVID19; sino, como demandaba ya antes de esta situación Pérez-Gómez (2019): "diseñar y organizar el espacio, el tiempo, las relaciones sociales, las actividades, el currículum y la evaluación para ayudar a formar el ciudadano culto, solidario y autónomo que exige la complejidad de este escenario global y digital contemporáneo" (p.4).

Desde hace unos años, la tecnología se ha considerado un facilitador de la educación, sin embargo, debido a las circunstancias, hemos vivido una situación en que la educación depende enteramente de la tecnología (Mateo-Berganza \& Lee, 2020). Durante el confinamiento acaecido en España entre los meses de marzo y junio de 2020, el empleo de los diferentes recursos tecnológicos (campus virtuales, foros de debate, videoconferencias, etc.) ha sido de gran ayuda para superar el curso 2019-2020. No obstante, la disponibilidad de dichos recursos no ha sido el único problema ya que ni docentes, debido en parte a la falta de experiencia e inseguridad que la no presencialidad ha generado en ellos (Cabero \& Valencia-Ortiz, 2021); ni discentes, que no han sido capaces de organizarse y se han sentido sobrecargados, se encontraban preparados para una situación similar.

El empleo de videotutoriales en la era post COVID19: valoración e influencia en la identidad docente del futuro profesional. Hernández-Ramos, J.P., Martínez-Abad, F., Sánchez-Prieto, J.C. 
En este caso, situándonos en la Universidad de Salamanca, concretamente en la asignatura de Metodología de Investigación Educativa/Socioeducativa, impartida en las titulaciones de Educación Social, Magisterio Educación Primaria, Magisterio Educación Infantil y Pedagogía, uno de los recursos más empleados por el profesorado, tanto para el desarrollo de contenidos teóricos como prácticos, ha sido el videotutorial (Van der Meij \& van der Meij, 2014; Karma et al., 2019), permitiendo a los estudiantes seguir las explicaciones del docente de manera asíncrona y repetir la visualización tantas ocasiones como considere necesarias.

De cara a la planificación de los próximos cursos, para no agravar en mayor medida en dicho estado de incertidumbre con inseguridades metodológicas, es necesario evaluar las prácticas docentes realizadas. Por ello, la finalidad de este estudio es doble. Por un lado, valorar, en base a las opiniones de los estudiantes, el empleo de videotutoriales como herramienta formativa en docencia virtual, concretamente durante el estado de alarma vivido en España entre los meses de marzo y junio de 2020. Por otro, a tenor de que los protagonistas del proceso formativo son futuros profesionales de la educación, es relevante destacar la influencia de las acciones del profesorado en la concepción docente de los estudiantes.

Para concretar estos aspectos, se plantean las siguientes cuestiones de investigación a las que se pretende ofrecer respuesta: ¿Cómo valoran los estudiantes universitarios el empleo de videotutoriales como herramienta formativa en enseñanza virtual? y ¿en qué medida influye en la creación de la identidad docente del futuro profesional de la educación la metodología del profesorado?

Con el objetivo de dar respuesta a estas preguntas, se propone un artículo dividido en cuatro secciones. En la primera se presenta un marco teórico en el que se describen los conceptos clave y antecedentes principales relacionados con el uso educativo de videotutoriales y la formación de la identidad docente. En la segunda sección, se describe la metodología empleada en esta investigación incluyendo el diseño de investigación, las variables incluidas y la escala empleada. En la sección tres se exponen los resultados obtenidos y, finalmente, en la sección cuatro se exploran las implicaciones de estos resultados, las limitaciones del estudio y las posibles líneas futuras de investigación.

\section{MARCO TEÓRICO}

Como hemos visto, las restricciones impuestas por el COVID-19 a la docencia presencial en educación superior han hecho que muchos docentes tengan que emplear diferentes herramientas para realizar el cambio a la docencia virtual (Cahapai, 2020). Entre los recursos empleados por los profesores durante este periodo se encuentra el uso de videotutoriales para la explicación asíncrona de contenidos a los estudiantes (Reimers \& Schleicher, 2020).

El uso de videotutoriales ya era una práctica presente en contextos de educación superior como un elemento de apoyo tanto a la docencia presencial (Lai et al., 2017) como a la semipresencial (Karma et al., 2019) y la no presencial (Scagnoli et al., 2017).

Entre sus principales ventajas destaca su capacidad para presentar la información empleando diferentes modalidades, auditiva y visual, que se refuerzan y complementan, así como el ofrecer un ejemplo del proceso que resulta fácil de seguir y que los estudiantes pueden replicar de manera autónoma (Van der Meij \& van der Meij, 2014).

El empleo de videotutoriales en la era post COVID19: valoración e influencia en la identidad docente del futuro profesional. Hernández-Ramos, J.P., Martínez-Abad, F., Sánchez-Prieto, J.C. 
Adicionalmente, el empleo de estos recursos también tiene un efecto positivo sobre la motivación de los estudiantes, lo que resulta especialmente importante en materias que pueden resultar poco atractivas (Martínez-Abad \& Hernández-Ramos, 2017). Estas características hacen que los videotutoriales sean empleados con frecuencia en diseños de clase invertida para guiar el proceso de autoaprendizaje del alumno (MurilloZamorano et al., 2019).

En consecuencia, el número de investigaciones acerca del uso educativo de los videotutoriales es cada vez mayor. Estas se pueden clasificar en dos grupos:

- Investigaciones centradas en el rendimiento académico: que confirman el impacto positivo en el desempeño de los estudiantes (ej. Kazanidis et al., 2019).

- Investigaciones centradas en la adopción: principalmente enfocadas en el análisis de la opinión y satisfacción de los estudiantes con estas herramientas (ej. Maziriri et al., 2020).

El contexto social generado por la pandemia en España permite analizar la eficacia de esta tecnología en un momento excepcional en el que la motivación y el autoaprendizaje han jugado un papel fundamental en el desarrollo del proceso de enseñanza.

Esta investigación pretende sacar partido de esta circunstancia para contribuir al desarrollo de la literatura sobre este tema, analizando la opinión de los estudiantes de carreras relacionadas con la educación sobre el uso de videotutoriales para explicar contenidos de estadística dentro de la asignatura de metodología de investigación educativa. En este sentido, hay que considerar que el uso de una determinada metodología educativa para la formación de futuros docentes no solo tiene un impacto sobre el rendimiento académico, sino que también contribuye a la construcción de su identidad docente (Hong et al., 2016).

La identidad docente se entiende como un proceso continuo y abierto que implica la interpretación y reinterpretación de las creencias del sujeto sobre la enseñanza y que se manifiesta en su práctica profesional, sus sentimientos de pertenencia a este colectivo y sus experiencias de aprendizaje (Dasa \& Derose, 2017). Esta identidad se compone de tres dimensiones (Monereo et al., 2013):

- Concepciones sobre el rol del profesional y los procesos de enseñanza: Hace referencia a las creencias que tiene el docente sobre cuál es su propia función profesional, cuáles son sus competencias principales y cómo debe ser el proceso de enseñanza aprendizaje.

- Metodologías de enseñanza, evaluación y gestión de incidentes críticos: Incluye los conocimientos sobre las distintas metodologías didácticas así como a las diferentes estrategias para la solución de conflictos: reactivas, de evitación o reflexivas (Martín et al., 2000).

- Emociones y sentimientos asociados a la práctica docente: Esta dimensión agrupa los sentimientos de satisfacción o insatisfacción que siente el docente hacia su profesión. En el caso de los educadores en formación además engloba al sentimiento de stress ante la futura práctica profesional.

En este sentido, la formación que el docente recibe durante sus años universitarios juega un papel esencial en la construcción de su identidad profesional, dado que se trata de un periodo en el que el sujeto realiza una intensa reflexión sobre su futura práctica

El empleo de videotutoriales en la era post COVID19: valoración e influencia en la identidad docente del futuro profesional. Hernández-Ramos, J.P., Martínez-Abad, F., Sánchez-Prieto, J.C. 
laboral y adquiere el conocimiento necesario para llevarla a cabo. Como resultado, al final de esta etapa los individuos abandonan el rol de estudiantes expertos para adoptar el de educadores novatos, lo que implica cambios en las tres dimensiones anteriormente mencionadas (So et al., 2010; Monereo et al., 2013).

Aunque el número de estudios centrados en el proceso de formación de la identidad docente y el interés de la comunidad científica es cada vez mayor (Izadina, 2014), el conocimiento que tenemos sobre este tema es todavía muy escaso, siendo necesario todavía profundizar más en esta cuestión (Horvath et al., 2018).

A este respecto, se puede observar cómo existe un mayor número de estudios centrados en cómo afecta a los educadores en formación el periodo de prácticas realizado en el último año de sus estudios universitarios (Yuan et al., 2019, Henry, 2016), en ocasiones explorando cómo éste modifica su identidad profesional y ésta a su vez influye en aspectos como su decisión de seguir adelante con su carrera (Hong et al., 2017).

Estos estudios, por tanto, están enfocados en la identidad docente de los estudiantes que se encuentran al final de su periodo de formación, siendo más escaso el número de estudios que analiza el efecto de la formación recibida en los años previos (Kavanoz et al., 2017). En estas investigaciones se plantea que los mecanismos mediante los que se conforma la identidad docente se encuentra el del modelado que hace referencia al proceso mediante el cual los estudiantes van adquiriendo una serie de concepciones sobre cómo debe ser la enseñanza y el tipo de profesionales que aspiran a ser a través de su experiencia como discentes y la observación los profesores que les han educado (Holt-Reynolds, 1992).

De esta manera, la participación en actividades que incorporan el uso de TIC como estudiantes puede condicionar su intención de utilizarlas en su futura práctica profesional (Sang et al., 2010 y Sánchez-Prieto et al., 2019).

Aunque como se ha expuesto, la percepción de los estudiantes sobre la eficacia del uso de videotutoriales como herramienta educativa ya ha sido explorada con anterioridad en la literatura (Maziriri et al., 2020), la relación entre estas percepciones y la formación identidad docente de los estudiantes de carreras educativas todavía se encuentra poco explorada. Es por eso que la investigación recogida en este artículo pretende contribuir a llenar este hueco en la literatura, planteándose el siguiente objetivo general: Estudiar la valoración que estudiantes universitarios de Grado en Ciencias de la Educación realizan del uso de videotutoriales como alternativa a las sesiones expositivas en la enseñanza no presencial, analizando la relación existente entre esta valoración y la intención de los estudiantes de incorporar este recurso en su futuro profesional.

\section{METODOLOGÍA}

Con el fin de alcanzar el objetivo y las cuestiones planteadas en este trabajo, se planteó un estudio de carácter cuantitativo puro (Johnson et al., 2007) a partir de un diseño de investigación cuasi-experimental solo postest de un solo grupo (Campbell \& Stanley, 1963). Así, se analizó la realidad tras la aplicación de una intervención educativa, o tratamiento, que consistió en el diseño y empleo de videotutoriales para la transmisión de los contenidos teóricos y prácticos de asignaturas de Metodología de Investigación en el ámbito de las Ciencias de la Educación. Debido al bajo nivel de experimentalidad de esta investigación, y que no se aplica una evaluación longitudinal pretest-postest, no

El empleo de videotutoriales en la era post COVID19: valoración e influencia en la identidad docente del futuro profesional. Hernández-Ramos, J.P., Martínez-Abad, F., Sánchez-Prieto, J.C. 
se establecen hipótesis de trabajo como tal, sino que se pretenden abordar las cuestiones de investigación planteadas en la introducción.

\subsection{Población y muestra}

Tras la definición de la población del estudio como el conjunto de estudiantes universitarios de titulaciones de Grado en Ciencias de la Educación, se obtuvo una muestra no probabilística por accesibilidad de un total de 240 estudiantes de los Grados en Pedagogía, Educación Social, Maestro en Educación Primaria y Maestro en Educación Infantil en una universidad pública de Castilla y León.

La distribución de la muestra por titulación fue de un 43.8\% de estudiantes de Magisterio en Educación Primaria, un $23.3 \%$ de estudiantes de Pedagogía, un $21.7 \%$ de estudiantes de Educación Social y un 11.3\% de estudiantes de Magisterio en Educación Infantil.

\subsection{Instrumento y variables}

Se empleó el cuestionario como instrumento de recogida de información. El cuestionario utilizado, adaptado de estudios previos relativos al empleo de TIC en la enseñanza universitaria (Martínez-Abad \& Hernández-Ramos, 2017; Olmos, et al., 2014), incluyó 11 ítems de escala de respuesta tipo Likert con 5 niveles de respuesta (totalmente en desacuerdo, en desacuerdo, ni de acuerdo ni en desacuerdo, de acuerdo y totalmente de acuerdo). En concreto, se trata de un cuestionario de autoeficacia que evalúa el nivel de competencias adquirido gracias al apoyo del tratamiento implementado. Cabe destacar al respecto que la literatura científica muestra evidencias sólidas del poder predictivo de las medidas de autoeficacia (Bandura \& Locke, 2003) en la investigación en Ciencias Sociales. La tabla 1 muestra los ítems del cuestionario.

\begin{tabular}{|l|l|}
\hline Escala & Enunciado ítem \\
\hline \multirow{5}{*}{ Ítems criterio } & $\begin{array}{l}\text { Si volviera a cursar la asignatura, me gustaría que se mantuviera el } \\
\text { empleo de videotutorial aunque fuera de forma presencial }\end{array}$ \\
\cline { 2 - 3 } & En el futuro emplearé videotutoriales en mi labor profesional \\
\hline $\begin{array}{l}\text { Escala } \\
\text { valoración } \\
\text { videotutoriales }\end{array}$ & $\begin{array}{l}1 \text { El empleo de videotutoriales desarrollar mi pensamiento crítico } \\
\text { alrededor de los contenidos de la materia }\end{array}$ \\
\cline { 2 - 3 } & $\begin{array}{l}2 \text { El empleo de videotutoriales me ha ayudado en la elaboración de } \\
\text { síntesis personales sobre los contenidos }\end{array}$ \\
\cline { 2 - 3 } & $\begin{array}{l}\text { 3 El empleo de videotutoriales me ha permitido generalizar los } \\
\text { contenidos teóricos a situaciones reales }\end{array}$ \\
\cline { 2 - 3 } & $\begin{array}{l}4 \text { El empleo de videotutoriales me ha ayudado a resolver problemas } \\
\text { prácticos }\end{array}$ \\
\cline { 2 - 3 } & $\begin{array}{l}5 \text { El empleo de videotutoriales me ha facilitado el análisis y la } \\
\text { reflexión sobre los contenidos estudiados }\end{array}$ \\
\hline
\end{tabular}

El empleo de videotutoriales en la era post COVID19: valoración e influencia en la identidad docente del futuro profesional. Hernández-Ramos, J.P., Martínez-Abad, F., Sánchez-Prieto, J.C. 
RED. Revista de Educación a Distancia. Núm. 65, Vol. 21. Artíc. 11, 08-01-2021

DOI: https://doi.org/10.6018/red.449321

\begin{tabular}{|l|l|}
\hline & $\begin{array}{l}6 \text { El empleo de videotutoriales ha facilitado la memorización de los } \\
\text { contenidos de la asignatura. }\end{array}$ \\
\cline { 2 - 3 } & $\begin{array}{l}7 \text { El empleo de videotutoriales me ha permitido emitir valoraciones } \\
\text { personales sobre los temas tratados }\end{array}$ \\
\hline $\begin{array}{l}\text { 8 El empleo de videotutoriales ha permitido autoevaluar mi } \\
\text { aprendizaje en la asignatura }\end{array}$ \\
\cline { 2 - 2 } $\begin{array}{l}9 \text { El empleo de videotutoriales me ha facilitado la organización del } \\
\text { estudio }\end{array}$ \\
\hline
\end{tabular}

Tabla 1. Ítems del instrumento aplicado

Dado que se plantea un estudio exploratorio, sin intención de establecer causalidad, se establecieron una serie de variables criterio y variables explicativas explicativas:

- Variables criterio: Referidos a la valoración global de los videotutoriales. Los dos ítems incluídos fueron extraídos de Martínez Abad \& Hernández Ramos (2017): Satisfacción general (Si volviera a cursar la asignatura, me gustaría que se mantuviera el empleo del videotutorial aunque fuera de forma presencial) e Intención futura de uso (En el futuro emplearé videotutoriales en mi labor profesional) en relación a la metodología basada en videotutoriales.

- Variables explicativas: Se incluyó una escala compuesta por 9 ítems, relativa a la valoración de los estudiantes sobre la utilidad de los videotutoriales en el proceso de enseñanza-aprendizaje. En concreto, se adaptaron los ítems de una de las dimensiones validadas en el estudio de Olmos et al. (2014) para la evaluación del uso de plataformas educativas en la enseñanza universitaria.

Dada la naturaleza ordinal de los ítems, de cara a analizar la fiabilidad y validez de la escala adaptada para este estudio (Olmos, 2014), se aplicó un análisis factorial exploratorio (mediante componentes principales) a partir de la matriz de correlaciones policórica (Martínez-Abad \& Rodríguez-Conde, 2017), comprobando la estructura unidimensional de la escala. Para ello se empleó el paquete de SPSS R-factor (Basto \& Pereira, 2012). Los resultados mostraron una estructura factorial unidimensional nítida, alcanzando el primer factor un $62.10 \%$ de varianza explicada, y todos los ítems una carga superior a 0.68 en el factor. Se obtienen igualmente buenos índices de ajuste del modelo ( $\mathrm{GFI}=.987, \mathrm{AGFI}=.978$, RMSR=.073). En cuanto al análisis de fiabilidad, la escala alcanzó un valor del alfa de Cronbach muy satisfactorio (.923), siendo la correlación ítem-total corregida superior a .6 en todos los ítems.

\subsection{Tratamiento: diseño e implementación de los videotutoriales}

En base a que tanto el Plan de Estudios de las asignaturas de Metodología de Investigación, como las necesidades concretas de los estudiantes de Pedagogía, Educación Social y Magisterio en Educación Infantil y Primaria son diversas; en cada una de las titulaciones los profesores implicados, atendiendo a las características y necesidades de sus estudiantes, diseñaron diferentes estrategias didácticas basadas en el empleo de videotutoriales.

El empleo de videotutoriales en la era post COVID19: valoración e influencia en la identidad docente del futuro profesional. Hernández-Ramos, J.P., Martínez-Abad, F., Sánchez-Prieto, J.C. 
En todo caso, de cara a mantener un estándar de comparabilidad y equidad entre los grupos, se establecieron unos criterios comunes para la elaboración de los materiales e implementación del tratamiento (Martínez-Abad \& Hernández-Ramos, 2017):

- En cada asignatura se elaboran diferentes videotutoriales: orientados a la exposición teórica de los contenidos, a mostrar y resolver ejercicios y a resolver dudas, tanto de contenidos teóricos como prácticos.

- De cara a facilitar la atención de los estudiantes durante todo el contenido, se intenta que los vídeos tengan la mínima duración posible, siendo requisito imprescindible la planificación previa por parte del profesor.

- Regularmente, los estudiantes tienen acceso a los vídeos correspondientes a los contenidos y actividades a trabajar siguiendo una programación previa en coherencia con el cronograma de actividades semanales.

- De manera complementaria, se emplean pequeños videotutoriales destinados a solucionar dudas concretas de alumnos o grupos de trabajo.

\subsection{Procedimiento y análisis de datos}

Los cuestionarios fueron implementados en la plataforma Google Forms y facilitados a los estudiantes a través de las asignaturas del Campus Virtual, que los contestaron de modo voluntario entre el 20 y 30 de mayo de 2020. En todo caso, en la recogida y tratamiento de la información se siguieron los estándares y principios éticos habituales (British Educarional Research Association, 2018).

Tras la recogida de la información, esta fue extraída de Google Forms y filtrada para su empleo con el paquete estadístico SPSS v.24. Tras confirmar la validez y fiabilidad de la escala empleada, se aplicaron análisis de datos de carácter descriptivo y correlacional. En el análisis de la significación de los contrastes de hipótesis (Chicuadrado y coeficientes de correlación) se estableció un nivel de significación del 5\%. La intensidad de los coeficientes de correlación se interpretó siguiendo los criterios propuestos por Cohen (1969).

\section{RESULTADOS}

\subsection{Valoraciones del empleo de videotutoriales}

A continuación, se exponen los resultados obtenidos, diferenciando dos apartados. En primer lugar, se muestran las valoraciones realizadas por los estudiantes a la hora de valorar el empleo de videotutoriales en la formación virtual durante el confinamiento; analizando en un primer momento los resultados obtenidos en los 9 ítems de la escala, para posteriormente centrarse en las dos cuestiones de valoración global.

El empleo de videotutoriales en la era post COVID19: valoración e influencia en la identidad docente del futuro profesional. Hernández-Ramos, J.P., Martínez-Abad, F., Sánchez-Prieto, J.C. 
Tabla 2. Valoración del empleo de videotutoriales en docencia virtual

\begin{tabular}{|c|c|c|c|c|c|c|c|c|}
\hline El empleo de videotutoriales... & Media & $\begin{array}{l}\text { Desv. } \\
\text { Tip. }\end{array}$ & $\begin{array}{c}1 \\
(\%)\end{array}$ & $\begin{array}{c}2 \\
(\%)\end{array}$ & $\begin{array}{l}3 \\
(\%)\end{array}$ & $\begin{array}{c}4 \\
(\%)\end{array}$ & $\begin{array}{c}5 \\
(\%)\end{array}$ & $\mathbf{N}$ \\
\hline $\begin{array}{l}\text {...me ha ayudado a desarrollar mi } \\
\text { pensamiento crítico alrededor de los } \\
\text { contenidos de la materia }\end{array}$ & 4,15 & ,758 & 0,8 & 0,8 & 14,6 & 49,6 & 34,2 & 240 \\
\hline $\begin{array}{l}\text {...me ha ayudado en la elaboración de } \\
\text { síntesis personales sobre los } \\
\text { contenidos }\end{array}$ & 4,37 & ,784 & 0,4 & 1,7 & 11,3 & 33,2 & 53,4 & 238 \\
\hline $\begin{array}{l}\text {...me ha permitido generalizar los } \\
\text { contenidos teóricos a situaciones reales }\end{array}$ & 4,14 & ,790 & 0,4 & 2,5 & 15,0 & 46,7 & 35,4 & 240 \\
\hline $\begin{array}{l}\text {...me ha ayudado a resolver problemas } \\
\text { prácticos }\end{array}$ & 4,60 & ,708 & 0,4 & 2,1 & 4,2 & 23,4 & 69,9 & 239 \\
\hline $\begin{array}{l}\text {...me ha facilitado el análisis y la } \\
\text { reflexión sobre los contenidos } \\
\text { estudiados }\end{array}$ & 4,45 & ,737 & 0,8 & 0,8 & 7,1 & 34,7 & 56,5 & 239 \\
\hline $\begin{array}{l}\text {...ha facilitado la memorización de los } \\
\text { contenidos de la asignatura. }\end{array}$ & 4,16 & 921 & 1,3 & 3,3 & 17,9 & 32,9 & 44,6 & 240 \\
\hline $\begin{array}{l}\text {...me ha permitido emitir valoraciones } \\
\text { personales sobre los temas tratados }\end{array}$ & 3,84 & ,873 & 0,8 & 5,0 & 27,1 & 43,3 & 23,8 & 240 \\
\hline $\begin{array}{l}\text {...ha permitido autoevaluar mi } \\
\text { aprendizaje en la asignatura }\end{array}$ & 4,01 & ,846 & 0,4 & 3,8 & 21,3 & 43,3 & 31,3 & 240 \\
\hline $\begin{array}{l}\text {...me ha facilitado la organización del } \\
\text { estudio }\end{array}$ & 4,29 & ,827 & 0,8 & 1,7 & 13,8 & 35,0 & 48,8 & 240 \\
\hline
\end{tabular}

En la tabla 2 quedan recogidas las respuestas obtenidas tras la aplicación de la escala de valoración a los sujetos de la muestra. Realizando una aproximación global inicial, la primera apreciación muestra un alto grado de aceptación por parte de los estudiantes, situándose el valor promedio en todas las cuestiones en un valor superior a $4 \mathrm{y}$ encontrándose la moda siempre en opciones de respuesta positivas.

Al profundizar en mayor medida en las valoraciones realizadas, se observa como las funcionalidades más destacadas por los estudiantes son resolver problemas prácticos (93,3\% de valoraciones positivas y $69,9 \%$ con la máxima puntuación), analizar y reflexionar sobre la materia $(91,2 \%$ de valoraciones positivas y $56,5 \%$ con la máxima puntuación) elaborar síntesis personales ( $86,6 \%$ de valoraciones positivas y $53,4 \%$ con la máxima puntuación) y organizar el estudio $(83,8 \%$ de valoraciones positivas y $48,8 \%$ con la máxima puntuación).

En contraposición a estos resultados, aunque a nivel global los resultados se han considerado como buenos, cabe destacar cierto grado de incertidumbre a la hora de valorar los videotutoriales como un recurso para realizar valoraciones personales sobre los temas tratados $(27,1 \%$ de indecisión y $5,8 \%$ de valoraciones negativas) 0 autoevaluar el proceso de aprendizaje (21,3\% de indecisión y 4,2\% de valoraciones negativas).

El empleo de videotutoriales en la era post COVID19: valoración e influencia en la identidad docente del futuro profesional. Hernández-Ramos, J.P., Martínez-Abad, F., Sánchez-Prieto, J.C. 
Para complementar los resultados obtenidos en la escala de valoración del recurso, se procede a analizar las respuestas obtenidas en las dos preguntas de valoración global establecidas como variables criterio en el estudio correlacional posterior.

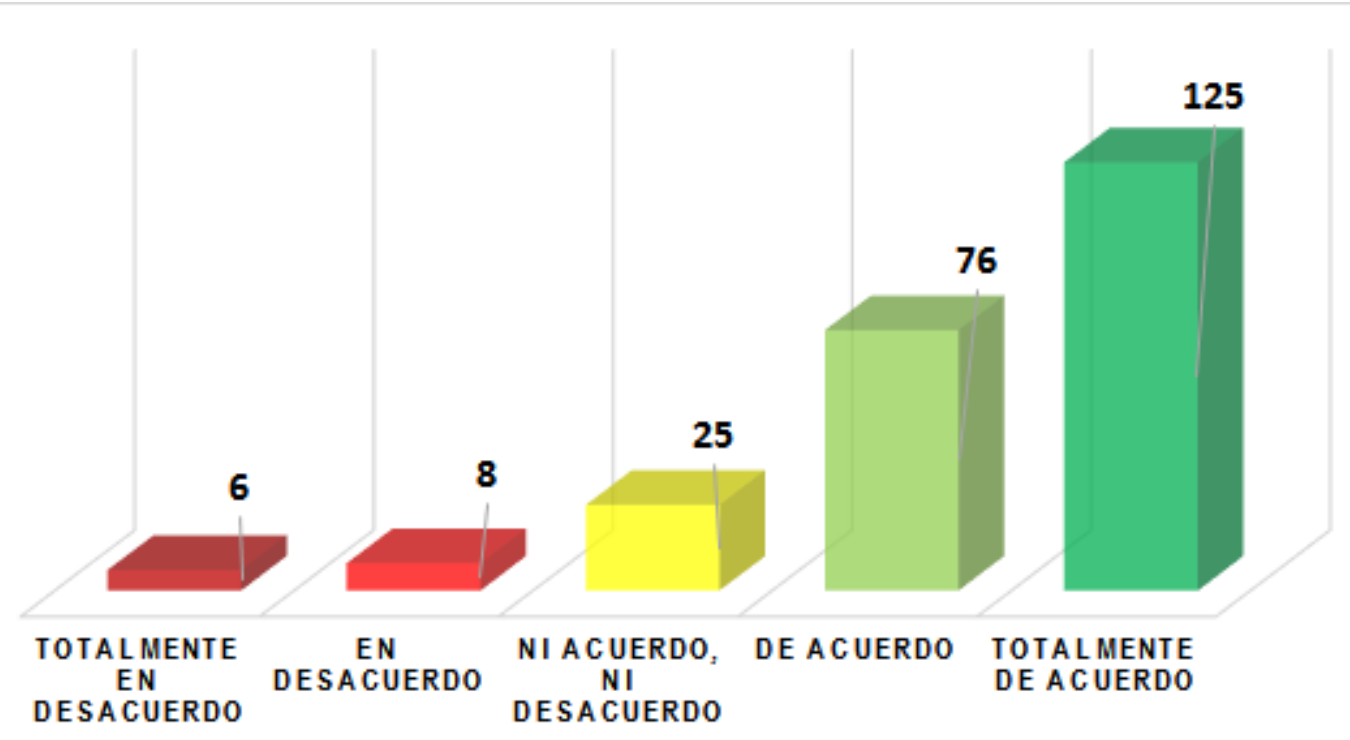

Figura 1: Empleo en cursos próximos.

En la Figura 1 queda recogida la respuesta de los estudiantes ante la pregunta: Si volviera a cursar la asignatura, me gustaría que se mantuviera el empleo de videotutorial aunque fuera de forma presencial. Las buenas valoraciones emitidas en las cuestiones previas confirman un buen nivel de aceptación de la herramienta y el $83,8 \%$ de los estudiantes (52,1\% totalmente de acuerdo y $31,7 \%$ de acuerdo) emiten una valoración positiva hacía la idea de que se mantenga el empleo del recurso por parte del profesorado. Así mismo, el 10,4\% de los estudiantes se manifiesta indiferente ante la cuestión; y el 5,8\% no consideran adecuada la posibilidad (3,3\% en desacuerdo y $2,5 \%$ totalmente en desacuerdo).

La segunda de las cuestiones de valoración global: En el futuro emplearé videotutoriales en mi labor profesional, es relevante porque la respuesta no solo se basa en el nivel de aceptación del recurso como alumno, sino que, al tratarse de futuros profesionales de la educación, influye en la respuesta del estudiante el grado de aceptación del recurso como futuro docente.

Los resultados obtenidos, reflejados en la figura 2, en coherencias tanto con las valoraciones emitidas en la escala como en la cuestión general previa, coinciden en mostrar una buena aceptación del empleo de videotutoriales como recurso educativo. Sin embargo, a pesar de que la proporción de consideraciones en contra (totalmente en desacuerdo + en desacuerdo) se mantiene, el número de estudiantes que manifiesta indiferencia aumenta considerablemente pasando de 25 a 71 . En lo relativo a las valoraciones positivas, se reducen considerablemente y la mayor parte de los futuros profesionales de la educación consideran que lo emplearán en un futuro, pero no están totalmente convencidos de ello.

El empleo de videotutoriales en la era post COVID19: valoración e influencia en la identidad docente del futuro profesional. Hernández-Ramos, J.P., Martínez-Abad, F., Sánchez-Prieto, J.C. 


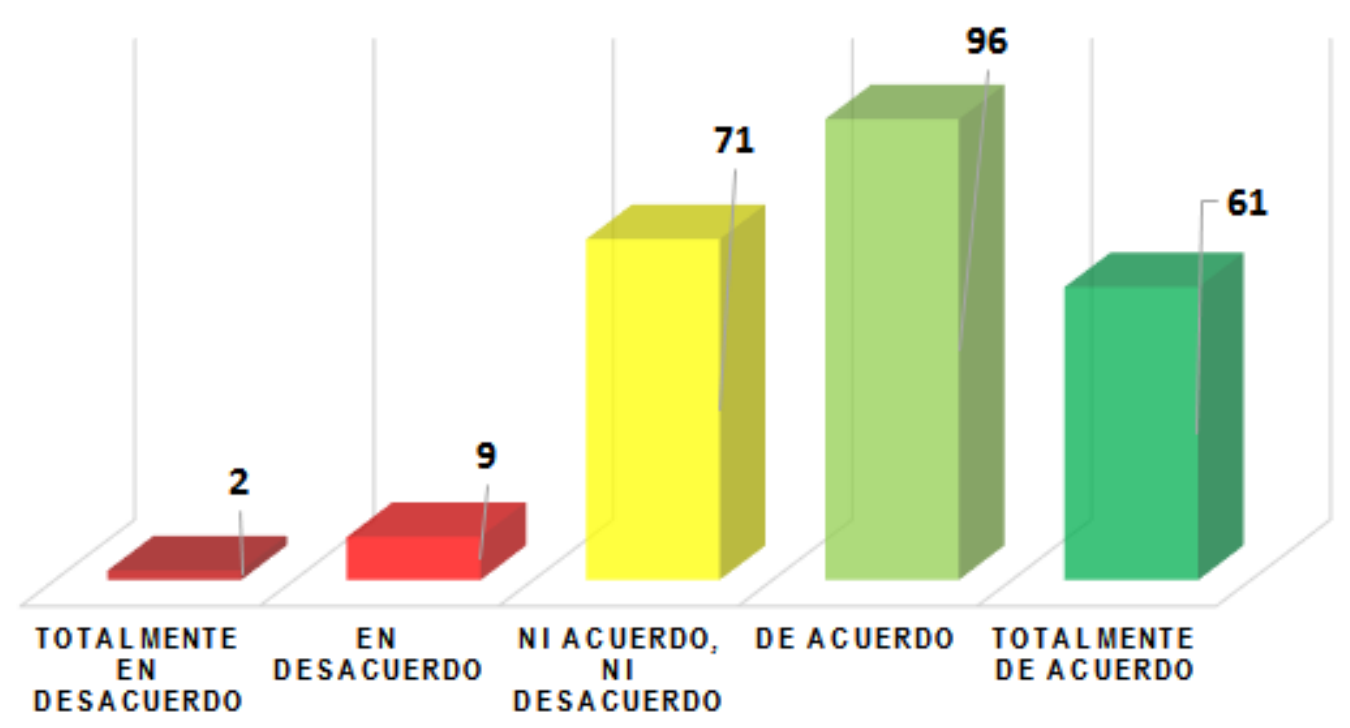

Figura 2. Intención empleo en el futuro profesional

Para complementar los resultados obtenidos en esta cuestión, cabe destacar que, a pesar de que las salidas profesionales de las diferentes titulaciones de la Facultad de Educación son diversas y el empleo de videotutoriales puede ser más oportuno en unos casos que otros, tras realizar la prueba de Chi Cuadrado no se encuentran diferencias proporcionales en base a la titulación del estudiante $\left(X^{2}=12.038\right.$; Sig. $\left.=.443\right)$. Así mismo, tampoco se encuentran diferencias $\left(X^{2}=3.867\right.$; Sig. $\left.=.424\right)$ si se comparan las opiniones de los estudiantes de las titulaciones más orientadas a la enseñanza formal (educación infantil y educación primaria) con los de las titulaciones enfocadas a la enseñanza no formal (Pedagogía y Educación social).

\subsection{Relación entre variables}

En la tabla 3 se pueden ver las correlaciones de Spearman obtenidas entre las variables criterio y los ítems de la escala de valoración de los videotutoriales. Mientras que en todos los casos se obtienen correlaciones altamente significativas, en base a los criterios de Cohen (1969), se observan a nivel general correlaciones con tamaños del efecto medios (entre .3 y .4), que en algunos casos llegan a medios-altos (superiores a .4). Parece, como era de esperar, que existe una mayor relación entre la satisfacción global del estudiante y la valoración de los mismos que con la percepción de uso futuro.

El empleo de videotutoriales en la era post COVID19: valoración e influencia en la identidad docente del futuro profesional. Hernández-Ramos, J.P., Martínez-Abad, F., Sánchez-Prieto, J.C. 
Tabla 3. Correlación de Spearman entre variables criterio y escala de valoración de los videotutoriales.

\begin{tabular}{|l|c|c|}
\hline & $\begin{array}{c}\text { Satisfacción } \\
\text { global }\end{array}$ & $\begin{array}{c}\text { Utilización } \\
\text { futura }\end{array}$ \\
\hline $\begin{array}{l}\text { 1 El empleo de videotutoriales desarrollar mi } \\
\text { pensamiento crítico alrededor de los contenidos } \\
\text { de la materia }\end{array}$ &, $319^{*}$ &, $285^{*}$ \\
\hline $\begin{array}{l}2 \text { El empleo de videotutoriales me ha ayudado } \\
\text { en la elaboración de síntesis personales sobre } \\
\text { los contenidos }\end{array}$ &, $337^{*}$ &, $257^{*}$ \\
\hline $\begin{array}{l}\text { 3 El empleo de videotutoriales me ha permitido } \\
\text { generalizar los contenidos teóricos a situaciones } \\
\text { reales }\end{array}$ &, $392^{*}$ &, $302^{*}$ \\
\hline $\begin{array}{l}4 \text { El empleo de videotutoriales me ha ayudado a } \\
\text { resolver problemas prácticos }\end{array}$ &, $444^{*}$ &, $374^{*}$ \\
\hline $\begin{array}{l}5 \text { El empleo de videotutoriales me ha facilitado } \\
\text { el análisis y la reflexión sobre los contenidos } \\
\text { estudiados }\end{array}$ &, $442^{*}$ &, $346^{*}$ \\
\hline $\begin{array}{l}\text { 6 El empleo de videotutoriales ha facilitado la } \\
\text { memorización de los contenidos de la } \\
\text { asignatura. }\end{array}$ &, $413^{*}$ &, $412^{*}$ \\
\hline $\begin{array}{l}7 \text { El empleo de videotutoriales me ha permitido } \\
\text { emitir valoraciones personales sobre los temas } \\
\text { tratados }\end{array}$ &, $390^{*}$ &, $463^{*}$ \\
\hline $\begin{array}{l}\text { 8 El empleo de videotutoriales ha permitido } \\
\text { autoevaluar mi aprendizaje en la asignatura }\end{array}$ &, $293^{*}$ &, $412^{*}$ \\
\hline $\begin{array}{l}9 \text { El empleo de videotutoriales me ha facilitado } \\
\text { la organización del estudio }\end{array}$ &, $442^{*}$ &, $386^{*}$ \\
\hline
\end{tabular}

En el caso del uso futuro, mientras que se observan correlaciones de tamaño medioalto principalmente con los ítems relacionados con cuestiones operativas del proceso de enseñanza y de adquisición de contenidos (memorización, autoevaluación o organización del estudio), se obtienen correlaciones de tamaño medio-bajo con los ítems asociados a cuestiones de aprendizaje, comprensión y adquisición de competencias (pensamiento crítico, elaboración de síntesis o generalización de los contenidos a la realidad). Estos resultados indican que los estudiantes universitarios valoran en mayor medida la utilidad de estas herramientas para la enseñanza de contenidos que su potencial para mejorar el aprendizaje y adquisición de competencias.

Por lo tanto, las evidencias recogidas sugieren que la identidad docente de los futuros profesionales del ámbito educativo se mantiene anclada en un paradigma basado en los contenidos y en la enseñanza, más que en un enfoque orientado hacia el aprendizaje y la adquisición de competencias.

Finalmente, a nivel general, mientras que la correlación obtenida entre ambas variables criterio alcanzó un valor de .529 (tamaño del efecto alto), la correlación entre la puntuación total en la escala aplicada y las variables satisfacción y uso futuro fue, respectivamente, de .497 y .488. Estos datos informan sobre la validez criterial y teórica del instrumento aplicado al mostrarse, conforme a la teoría de la acción razonada (Ajzen \& Fishbein, 1980) una clara relación entre la percepción de utilidad del videotutorial y la intención de uso futuro.

El empleo de videotutoriales en la era post COVID19: valoración e influencia en la identidad docente del futuro profesional. Hernández-Ramos, J.P., Martínez-Abad, F., Sánchez-Prieto, J.C. 


\section{DISCUSIÓN Y CONCLUSIONES}

En el momento en que el estado de la alarma obliga a todo el sistema educativo a adaptarse a una enseñanza a distancia, las primeras alertas se centraban en la falta de recursos tecnológicos en los hogares de profesores y alumnos, agravándose este problema en mayor medida en las zonas rurales donde en muchas ocasiones la conexión a internet es deficitaria. Ante estos problemas, como docentes, nuestras aportaciones están muy condicionadas y en ocasiones se limitan a apoyar las diferentes medidas y ayudas tomadas por las administraciones. Sin embargo, como reflejan Cabero y Valencia-Ortiz (2021): "la brecha digital no solo se refiere al acceso a las tecnologías, sino también al conocimiento de ellas, es decir, a qué somos capaces de hacer con las tecnologías y para qué" (p.119), siendo en ese aspecto en el que se centra el presente estudio.

A nivel general, los resultados de este trabajo pueden resultar valiosos para la comunidad docente universitaria y no universitaria, al mostrar tanto una alternativa como un complemento al desarrollo de las sesiones expositivas síncronas (presenciales o virtuales) que permite una mayor flexibilidad y control en la planificación del proceso de enseñanza-aprendizaje. Las evidencias obtenidas indican una alta satisfacción general por parte del alumnado, principalmente en cuanto al potencial de esta metodología para la resolución y comprensión de los contenidos prácticos, facilitando la reflexión y elaboración de síntesis personales. Cabe destacar inicialmente esta cuestión, ya que la organización de prácticas y actividades de trabajo en grupo es uno de los aspectos en que se están detectando un mayor número de inconvenientes a la hora de adaptar la presencialidad a otras modalidades formativas (Allen et al., 2020; García-Peñalvo et al., 2020). Así mismo, las valoraciones recogidas manifiestan que el empleo de videotutoriales en la enseñanza universitaria fomenta y facilita la reflexión, el análisis y la comprensión de los contenidos de la materia, habilidades necesarias y prioritarias en un sistema educativo basado en el desarrollo de competencias.

A lo largo del trabajo se ha expuesto cómo los estudiantes, poco acostumbrados a una docencia no presencial, se han visto en ocasiones sobrepasados. Del mismo modo, muchos docentes han vivido situaciones similares al aumentar su carga de trabajo (Allen, Rowan y Singh, 2020), siendo necesario replantear no solo los contenidos de las asignaturas (García-Planas \& Torres, 2021) sino también las metodologías y los recursos empleados.

Por otro lado, este trabajo refleja con claridad la influencia de la metodología empleada en la formación de la identidad docente de los estudiantes. Los resultados obtenidos evidencian la correlación existente entre la satisfacción de los estudiantes con el uso de videotutoriales y su intención de utilizarlos en su futura práctica profesional, lo que se sitúa en la línea de investigaciones previas (Holt-Reynolds, 1992; Kavanoz et al., 2017) confirmando la influencia de las experiencias como estudiantes en la formación del modelo docente asumido por los educandos.

En este sentido se puede comprobar cómo aquellos aspectos que tienen más relación con la intención de utilizar videotutoriales en la futura práctica docente son los que hacen referencia al autoaprendizaje (autoevaluación, organización, valoración y memorización), lo que parece apuntar que los futuros educadores se inclinan por un modelo educativo en el que se potencie la autonomía del estudiante y lo coloque en el centro del proceso (Van der Meij \& van der Meij, 2014). Estos aspectos educativos son precisamente aquellos en los que incide con mayor énfasis el empleo de este tipo de

El empleo de videotutoriales en la era post COVID19: valoración e influencia en la identidad docente del futuro profesional. Hernández-Ramos, J.P., Martínez-Abad, F., Sánchez-Prieto, J.C. 
recursos, lo que puede indicar también una posible influencia por modelado en la identidad docente de los estudiantes que deberá ser explorada en mayor profundidad en futuras investigaciones.

Adicionalmente, llama especialmente la atención la alta correlación de la intención de uso futuro con el ítem referido a la memorización del contenido (.412). Esta evidencia sugiere que los estudiantes conciben el proceso de enseñanza-aprendizaje como una actividad en la que tiene mucho peso el aspecto memorístico, resultado coherente con el hecho de que los ítems que hacen referencia a la elaboración crítica de contenidos y la aplicación de los mismos a situaciones prácticas parecen ser los que tienen una relación menor con la intención de uso de videotutoriales en su futura profesión. Cabe plantearse, por tanto, si esta baja correlación se debe a limitaciones propias de este recurso o si son resultado del diseño didáctico empleado en las asignaturas. La respuesta a esta cuestión constituye también una interesante línea de investigación.

La presente investigación, por tanto, realiza una contribución valiosa en el ámbito de la docencia universitaria en un contexto incierto como el actual. La alta satisfacción de los estudiantes con la integración de los videotutoriales en los procesos formativos universitarios aporta evidencias sobre el potencial educativo de este recurso, tanto en contextos de aprendizaje híbridos como en entornos no presenciales. Más concretamente, parece que el empleo de videotutoriales es especialmente útil en el desarrollo de las prácticas, ya que facilitan al estudiante la generalización de los contenidos teóricos a casos prácticos y la reflexión y elaboración de síntesis personales. Esta cuestión es de especial relevancia, al encontrarnos en una situación de profunda preocupación y debate en la comunidad universitaria en torno a la adaptación de las prácticas de las materias a un modelo semipresencial e incluso no presencial.

Este trabajo también realiza un aporte de carácter empírico en el ámbito del desarrollo de la identidad personal docente a partir de las experiencias como estudiante. El empleo de recursos como los videotutoriales, que centran el foco de la enseñanza en la actividad y aprendizaje del alumnado más que en el propio contenido o la labor del profesor, puede ejercer de reactivo en la transformación de la concepción de la actividad docente y de los procesos de enseñanza-aprendizaje por parte de la comunidad educativa. Es por eso que la integración de este tipo de recursos en los procesos educativos genera un valor añadido no solo al nivel micro del grupo-aula, sino también a niveles macro.

A pesar de las aportaciones que realiza este trabajo, hay que ser cautos con las conclusiones extraídas debido fundamentalmente a algunas limitaciones relacionadas con la instrumentación y el muestreo aplicados. En primer lugar, es importante destacar que la muestra obtenida está restringida a estudiantes de Ciencias de la Educación de una universidad pública de Castilla y León. Las características de esta muestra han facilitado el análisis de la relación entre la identidad docente de los futuros profesionales de la educación y su participación como estudiantes en procesos de enseñanzaaprendizaje que incorporan videotutoriales como recurso educativo. No obstante, la muestra seleccionada dificulta la generalización de resultados a la población general de estudiantes universitarios. Por otro lado, a pesar de que se adaptó un instrumento validado para la valoración de la satisfacción del alumnado con el empleo de los videotutoriales, la medición de la intención de uso futuro de este recurso se realizó con un único ítem, el cual se asocia a la configuración de la identidad docente del profesor. La generalización realizada, por tanto, se realizó a partir de información reducida.

El empleo de videotutoriales en la era post COVID19: valoración e influencia en la identidad docente del futuro profesional. Hernández-Ramos, J.P., Martínez-Abad, F., Sánchez-Prieto, J.C. 
Por todo ello, de cara a futuros estudios, creemos necesario realizar un esfuerzo en ampliar la población diana a estudiantes universitarios de otras áreas de conocimiento, de manera que se pueda realizar un estudio diferencial del potencial de este recurso. Igualmente, en la línea de estudios restringidos al profesorado y futuro profesorado, cabe incluir una instrumentación más extensa en relación a la construcción y desarrollo de la identidad docente que permita explorar en mayor profundidad las relaciones apuntadas por este estudio. Otras líneas secundarias que quedan abiertas gracias a los resultados aquí obtenidos tienen que ver con explorar si el uso de videotutoriales supone una sobrecarga o una liberación de trabajo para el profesor en un contexto de docencia híbrida o analizar más en profundidad las razones por las que una pequeña parte de los estudiantes consideran inadecuado el empleo de este recurso en la docencia universitaria.

En suma, de cara a reducir la citada brecha digital, estas conclusiones alcanzan especial relevancia en la situación actual de incertidumbre metodológica, al mostrar una opción válida y adecuada, tanto para contextos presenciales, no presenciales e incluso hídricos. No obstante, el empleo eficaz de videotutoriales en la docencia universitaria implica tanto el diseño técnico adecuado de los mismos como su correcta planificación e integración en los procesos de enseñanza-aprendizaje. Las organizaciones dedicadas a la formación del profesorado tienen el deber de adaptar sus enseñanzas a los contextos y necesidades vigentes, por lo que se evidencia la necesidad de incluir e incidir en estas cuestiones dentro de los procesos de formación y desarrollo profesional docente del profesorado y futuro profesorado. De hecho, cabe recordar que en el desarrollo de la identidad docente influyen no solo las experiencias como estudiante, sino también los procesos de formación inicial y permanente.

Presentación del artículo: 10 de noviembre de 2020 Fecha de aprobación: 29 de diciembre de 2020

Fecha de publicación: 08 de enero de 2021

Hernández-Ramos, J.P., Martínez-Abad, F., Sánchez-Prieto, J.C (2021). El empleo de videotutoriales en la era post COVID19: valoración e influencia en la identidad docente del futuro profesional. RED. Revista de Educación a distancia, 21(65). https://doi.org/10.6018/red.449321

\section{Financiación}

Este trabajo no ha recibido ninguna subvención específica de los organismos de financiación en los sectores públicos, comerciales o sin fines de lucro.

\section{Referencias bibliográficas.}

Allen, J., Rowan, L., \& Singh, P. (2020). Teaching and teacher education in the time of COVID-19. Asia-Pacific Journal of Teacher Education, 48(3), 233-236. https://doi.org/10.1080/1359866X.2020.1752051

Ajzen, I., \& Fishbein, M. (1980). Understanding attitudes and predicting social behavior. Prentice-Hall.

El empleo de videotutoriales en la era post COVID19: valoración e influencia en la identidad docente del futuro profesional. Hernández-Ramos, J.P., Martínez-Abad, F., Sánchez-Prieto, J.C. 
Basto, M., \& Pereira, J. M. (2012). An SPSS R-Menu for Ordinal Factor Analysis. Journal of statistical software, 46(4).

Cabero, J., \& Valencia-Ortiz, R. (2021). Y el COVID-19 transformó al sistema educativo: Reflexiones y experiencias por aprender. IJERI: International Journal of Educational Research and Innovation, 15, 217-227. https://doi.org/10.46661/ijeri.5246

Bandura, A., \& Locke, E. A. (2003). Negative self-efficacy and goal effects revisited. The Journal of Applied Psychology, 88(1), 87-99. https://doi.org/10.1037/00219010.88.1.87

British Educational Research Association. (2018). Ethical guidelines for educational research (fourth edition). https://www.bera.ac.uk/researchersresources/publications/ethical-guidelines-for-educational-research-2018

Cahapay, M. B. (2020). Rethinking Education in the New Normal Post-COVID-19 Era: A Curriculum Studies Perspective. Aquademia, 4(2), 1-5. https://doi.org/10.29333/aquademia/8315

Cohen, J. (1969). Statistical power analysis for the behavioral sciences. Academic Press.

Dassa, L. \&, Derose, D.S. (2017). Get in the Teacher Zone: A Perception Study of Preservice Teachers and Their Teacher Identity. Issues in Teacher Education, 26(1), 101-113.

García-Planas, M. I., \& Torres, J. T. (2021). Transición de la docencia presencial a la no presencial en la UPC durante la pandemia del COVID-19. IJERI: International Journal of Educational Research and Innovation, 15, 177-187. https://doi.org/10.46661/ijeri.5015

Henry, A. (2016). Conceptualizing Teacher Identity as a Complex Dynamic System. Journal of Teacher Education, 67(4), 291-305. doi:10.1177/0022487116655382

Holt-Reynolds, D. (1992). Personal History-Based Beliefs as Relevant Prior Knowledge in Course Work. American Educational Research Journal, 29(2), 325-349.

Hong, J., Greene, B., \& Lowery, J. (2016). Multiple dimensions of teacher identity development from pre-service to early years of teaching: a longitudinal study. Journal of Education for Teaching, 43(1), 84-98. http://doi.org/10.1080/02607476.2017.1251111

Hong, J., Greene, B., Roberson, R., Cross Francis, D., \& Rapacki Keenan, L. (2017). Variations in pre-service teachers' career exploration and commitment to teaching. Teacher Development, 22(3), 408-426. http://doi.org/10.1080/13664530.2017.1358661

Horvath, M., Goodell, J. E., \& Kosteas, V. D. (2018). Decisions to enter and continue in the teaching profession: Evidence from a sample of U.S. secondary STEM teacher candidates. Teaching and Teacher Education, 71, 57-65. http://doi.org/10.1016/j.tate.2017.12.007

El empleo de videotutoriales en la era post COVID19: valoración e influencia en la identidad docente del futuro profesional. Hernández-Ramos, J.P., Martínez-Abad, F., Sánchez-Prieto, J.C. 
Izadinia, M. (2014) Teacher educators' identity: a review of literature. European Journal of Teacher Education, 37(4), 426-441. http://doi.org/10.1080/02619768.2014.947025

Karma, I. G. M., Darma, I. K., \& Santiana, I. M. A. (2019). Teaching strategies and technology integration in developing blended learning of applied mathematics subject. International Research Journal of Engineering, IT \& Scientific Research, 5(5), 16-25. https://doi.org/10.21744/irjeis.v5n5.726

Kavanoz, S., Yüksel, H. G., \& Varol, B. (2017) Evolvement of pre-service language teachers' beliefs through teacher education. International Journal of Progressive Education, 13(1), 119-135.

Kazanidis, I., Pellas, N., Fotaris, P. and Tsinakos, A. (2019), Can the flipped classroom model improve students' academic performance and training satisfaction in Higher Education instructional media design courses?. British Journal of Educational Technologies, 50, 2014-2027. https://doi.org/10.1111/bjet.12694

Lai, G., Zhu, Z., \& Williams, D. (2017). Enhance Students' Learning in Business Statistics Class Using Video Tutorials. Journal of Teaching and Learning With Technology, 6(1), 31-44. https://doi.org/10.14434/jotlt.v6.n1.21161

Martín, M. D., Jiménez, M. P. \& Fernández-Aabascal, E. G. (2000). Estudio sobre la escala de estilos y estrategias de afrontamiento (E3A). Revista electrónica de motivación y emoción, 3(4). http://reme.uji.es/articulos/agarce4960806100/texto.html

Martínez-Abad, F., \& Rodríguez-Conde, M. J. (2017). Comportamiento de las correlaciones producto-momento y tetracórica-policórica en escalas ordinales: Un estudio de simulación. RELIEVE - Revista Electrónica de Investigación y Evaluación Educativa, 23(2). https://doi.org/10.7203/relieve.23.2.9476

Martínez-Abad, F., \& Hernández-Ramos, J. P. (2017). Flipped Classroom con píldoras audiovisuales en prácticas de análisis de datos para la docencia universitaria: Percepción de los estudiantes sobre su eficacia. En S. Pérez Aldeguer, G. Castellano Pérez, \& A. Pina Calafi (Eds.), Propuestas de Innovación Educativa en la Sociedad de la Información (pp. 92-105). Adaya Press. https://dialnet.unirioja.es/servlet/articulo?codigo=6122816

Mateo-Berganza, M., \& Lee, C. (2020). Una revolución silenciosa. En M. MateoBerganza \& C. Lee (Eds.), Tecnología: Lo que puede y no puede hacer por la educación. Una comparación de cinco historias de éxito. (pp. 20-33). Banco Iberoamericano de desarrollo. http://dx.doi.org/10.18235/0002401

Maziriri, E.T., Gapa, P., \& Chuchu, T. (2020). Student Perceptions towards the Use of YouTube as an Educational Tool for Learning and Tutorials. International Journal of Instruction, 13(2), 119-138. https://doi.org/10.29333/iji.2020.1329a

Monereo, C., Weise, C., \& Alvarez, I. (2013). Cambiar la identidad docente en la universidad. Formación basada en incidentes dramatizados. Infancia y Aprendizaje, 36(3), 323-340. http://doi.org/10.1174/021037013807533043

El empleo de videotutoriales en la era post COVID19: valoración e influencia en la identidad docente del futuro profesional. Hernández-Ramos, J.P., Martínez-Abad, F., Sánchez-Prieto, J.C. 
Murillo-Zamorano, L. R., López Sánchez, J. Á., \& Godoy-Caballero, A. L. (2019). How the flipped classroom affects knowledge, skills, and engagement in higher education: Effects on students' satisfaction. Computers \& Education, 141, 1-18. https://doi.org/10.1016/j.compedu.2019.103608

Olmos, S., Martínez-Abad, F., Torrecilla, E. M., \& Mena, J. J. (2014). Análisis psicométrico de una escala de percepción sobre la utilidad de Moodle en la universidad. RELIEVE - Revista Electrónica de Investigación y Evaluación Educativa, 20(2), art. 1. https://doi.org/10.7203/relieve.20.2.4221

Palazon-Herrera, J. (2018). Effectiveness and motivation towards the use of video tutorials when learning how to use music software. Cultura y Educacion, 30(4), 663692. https://doi.org/10.1080/11356405.2018.1514804

Pérez-Gómez, Á. I. (2019). Ser docente en tiempos de incertidumbre y perplejidad. Márgenes Revista de Educación de la Universidad de Málaga, 3-17. https://doi.org/10.24310/mgnmar.v0i0.6497

Reimers, F. M., \& Schleicher, A. (2020). A framework to guide an education response to the COVID-19 Pandemic of 2020.2 OECD. https://globaled.gse.harvard.edu/files/geii/files/framework_guide_v2.pdf

Sánchez-Prieto, J. C., Hernández-García, Á., García-Peñalvo, F. J., Chaparro-Peláez, J., \& Olmos-Migueláñez, S. (2019). Break the walls! Second-Order barriers and the acceptance of mLearning by first-year pre-service teachers. Computers in Human Behavior, 95, 158-167. https://doi.org/10.1016/j.chb.2019.01.019

Sang, G., Valcke, M., Braak, J.v., \& Tondeur, J. (2010). Student teachers' thinking processes and ICT integration: Predictors of prospective teaching behaviors with educational technology. Computers \& Education, 54(1), 103-112. https://doi.org/10.1016/j.compedu.2009.07.010

Scagnoli, N. I., McKinney, A., \& Moore-Reynen, J. (2017). Video lectures in eLearning. In F. Nafukho, \& B. Irby (Eds.) Handbook of research on innovative technology integration in higher education (pp.115-134). IGI Global.

So, H., Choi, H., Lim, W.Y., \& Xiong, Y. (2012). Little experience with ICT: Are they really the Net Generation student-teachers? Computers \& Education, 59(4), 1234-1245.

Trujillo-Sáez, F., Fernández-Navas, M., Montes-Rodríguez, M., Segura-Robles, A., Alaminos-Romero, F. J., \& Postigo-Fuentes, A. Y. (2020). Panorama de la educación en España tras la pandemia de COVID-19: La opinión de la comunidad educativa. Fad. http://doi.org/10.5281/zenodo-3878844

Van der Meij, H., \& van der Meij, J. (2014). A comparison of paper-based and video tutorials for software learning. Computers \& Education, 78, 150-159. https://doi.org/10.1016/j.compedu.2014.06.003

Yuan, R., Liu, W., \& Lee, I. (2019). Confrontation, negotiation and agency: exploring the inner dynamics of student teacher identity transformation during teaching practicum. Teachers and Teaching, 25(8), 1-22. https://doi.org/10.1080/13540602.2019.1688286

El empleo de videotutoriales en la era post COVID19: valoración e influencia en la identidad docente del futuro profesional. Hernández-Ramos, J.P., Martínez-Abad, F., Sánchez-Prieto, J.C. 